\title{
Reduction of Adult Hippocampal Neurogenesis Confers Vulnerability in an Animal Model of Cocaine Addiction
}

\author{
Michele A. Noonan, ${ }^{1}$ Sarah E. Bulin, ${ }^{1}$ Dwain C. Fuller, ${ }^{2}$ and Amelia J. Eisch ${ }^{1}$ \\ ${ }^{1}$ Department of Psychiatry, University of Texas Southwestern Medical Center, Dallas, Texas 75390-9070, and ${ }^{2}$ Department of Pathology and Laboratory \\ Medicine Toxicology, Veterans Affairs North Texas Health Center, Dallas, Texas 75216
}

\begin{abstract}
Drugs of abuse dynamically regulate adult neurogenesis, which appears important for some types of learning and memory. Interestingly, a major site of adult neurogenesis, the hippocampus, is important in the formation of drug-context associations and in the mediation of drug-taking and drug-seeking behaviors in animal models of addiction. Correlative evidence suggests an inverse relationship between hippocampal neurogenesis and drug-taking or drug-seeking behaviors, but the lack of a causative link has made the relationship between adult-generated neurons and addiction unclear. We used rat intravenous cocaine self-administration in rodents, a clinically relevant animal model of addiction, to test the hypothesis that suppression of adult hippocampal neurogenesis enhances vulnerability to addiction and relapse. Suppression of adult hippocampal neurogenesis via cranial irradiation before drug-taking significantly increased cocaine self-administration on both fixed-ratio and progressive-ratio schedules, as well as induced a vertical shift in the dose-response curve. This was not a general enhancement of learning, motivation, or locomotion, because sucrose self-administration and locomotor activity were unchanged in irradiated rats. Suppression of adult hippocampal neurogenesis after drug-taking significantly enhanced resistance to extinction of drug-seeking behavior. These studies identify reduced adult hippocampal neurogenesis as a novel risk factor for addiction-related behaviors in an animal model of cocaine addiction. Furthermore, they suggest that therapeutics to specifically increase or stabilize adult hippocampal neurogenesis could aid in preventing initial addiction as well as future relapse.
\end{abstract}

\section{Introduction}

Drug addiction is an enormous problem, with $4.9 \%$ of the world population abusing drugs annually (United Nations Office on Drugs and Crime, 2008). Addiction is exceptionally challenging to prevent and treat, as underscored by the high propensity of addicts to relapse to drug-taking. For example, one study found that $44 \%$ of cocaine users relapsed on average $72 \mathrm{~d}$ after treatment (Hubbard and Marsden, 1986). A promising approach for addiction prevention and treatment is the identification of vulnerability factors. For example, rodent models of drug abuse have identified that stress (Miczek and Mutschler, 1996), high locomotor response to novelty (Piazza et al., 2000), and high levels of cocaine self-administration (Edwards et al., 2007) are addiction vulnerability factors, each meeting the criteria of producing a vertical shift in the dose-response curve and enhancing drugseeking (Piazza et al., 2000). However, because addiction is a complex disorder, much work is needed to comprehensively identify additional vulnerability factors.

Received Aug. 28, 2009; revised 0ct. 30, 2009; accepted Nov. 9, 2009.

This work was supported by National Institute of Drug Abuse Grants F31DA021045 (M.A.N.) and K02DA023555 and R01DA01676505 (A.J.E.). We thank Chandana Ravikumar for excellent technical assistance, Dr. Erin Larson for excellent advice, and Amy Arguello, Nathan DeCarolis, and Drs. Diane Lagace and Madeleine Johnson for very helpful discussions of this paper. We are grateful to National Institute on Drug Abuse for providing the cocaine used in these studies.

Correspondence should be addressed to Amelia J. Eisch, Department of Psychiatry, University of Texas Southwestern Medical Center, 5323 Harry Hines Boulevard, Dallas, TX 75390-9070. E-mail: amelia.eisch@ utsouthwestern.edu.

DOI:10.1523/JNEUROSCI.4256-09.2010

Copyright $\odot 2010$ the authors $\quad 0270-6474 / 10 / 300304-12 \$ 15.00 / 0$
The hippocampus is increasingly recognized as having a potential role in addiction and is implicated in drug-context memory (Meyers et al., 2006; Shen et al., 2006; Hernández-Rabaza et al., 2008) and relapse to drug-seeking (Vorel et al., 2001; Fuchs et al., 2005). One aspect of hippocampal plasticity that has not been thoroughly considered as a potential vulnerability factor for addiction is neurogenesis, or the ability of the dentate gyrus subgranular zone (SGZ) to give rise to new neurons throughout life (Eisch, 2002; Abrous et al., 2005). Clinically relevant selfadministration studies reveal dynamic regulation of adult hippocampal neurogenesis by myriad drugs of abuse, including cocaine (Eisch et al., 2000; Abrous et al., 2002; Mandyam et al., 2008; Noonan et al., 2008). Interestingly, levels of adult hippocampal neurogenesis are negatively correlated with drugtaking and drug-seeking behaviors: manipulations that increase adult hippocampal neurogenesis-environmental enrichment, chronic treatment with antidepressants, and exercise (van Praag et al., 1999; Malberg et al., 2000; Brown et al., 2003) — are associated with decreased drug-taking and relapse (Kanarek et al., 1995; Baker et al., 2001; Green et al., 2002; Stairs et al., 2006; Smith et al., 2008), whereas those that decrease neurogenesis-stress and schizophrenia (Mirescu and Gould, 2006; Reif et al., 2006) —are associated with increased drug-taking and relapse (Erb et al., 1996; Chambers and Self, 2002; Covington and Miczek, 2005). Together with data that reduced adult neurogenesis can lead to cognitive deficits (Snyder et al., 2005; Saxe et al., 2006; Dupret et al., 2008; Imayoshi et al., 2008), these correlative findings suggest the untested hypothesis that reduced hippocampal neurogenesis is a vulnerability factor for addiction. 
A Irradiation before cocaine self-administration (CSA)

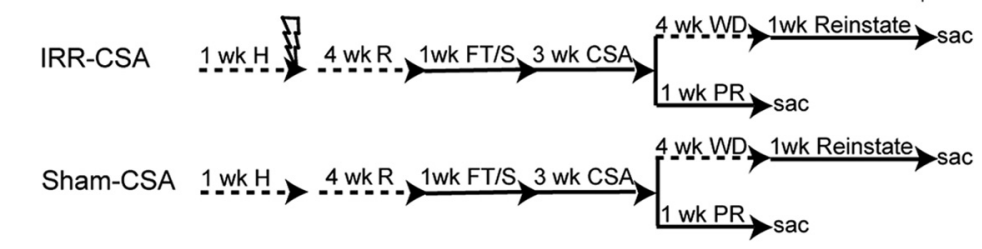

B Irradiation before sucrose self-administration (SSA)

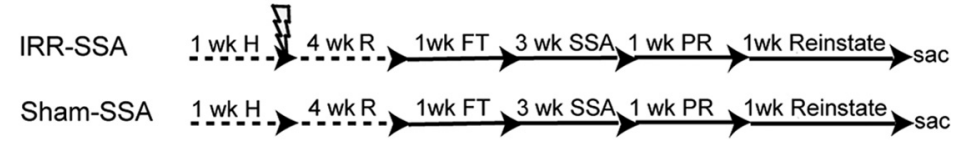

C Irradiation after cocaine self-administration

CSA-WD/IRR

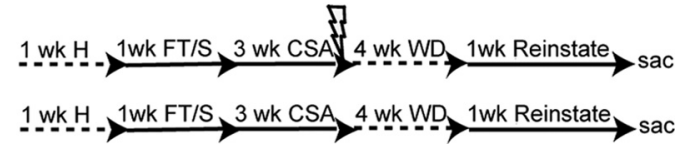

CSA-WD/Sham

Figure 1. Cranial irradiation decreases adult neurogenesis. A, IRR-CSA $(n=21)$ and Sham-CSA $(n=18)$ rats were habituated to the animal facility for 1 week, anesthetized ( $50 \mathrm{mg} / \mathrm{kg}$ sodium pentobarbital, i.p.), irradiated, and left in the home cage to recover for 4 weeks (with handling every $3 \mathrm{~d}$ ). Rats completed food training, were implanted with an intravenous catheter, and allowed to recover for several days. Three weeks of CSA followed. Rats were then split into groups for progressive-ratio testing (PR) or dose-response and reinstatement. Progressive-ratio rats had $6 \mathrm{~d}$ of testing and then were killed the next day (IRR, $n=7$; Sham, $n=5$ ). The remaining rats were given a dose-response test on the 16th day of CSA (IRR, $n=14$; Sham, $n=13)$ and then were placed in the home cage for 4 weeks of WD. Daily reinstatement testing followed, which consisted of an extinction session for at least $1 \mathrm{~h}$, followed by reinstatement testing to several variables as described in Materials and Methods: the context itself, the cue light, saline, intraperitoneal cocaine (5 or $15 \mathrm{mg} / \mathrm{kg}$ ), and finally footshock stress. A locomotor test (basal and after a $15 \mathrm{mg} / \mathrm{kg}$ cocaine injection) was done on the last day. Rats were killed $24 \mathrm{~h}$ later. $\boldsymbol{B}$, The experimental design for IRR-SSA $(n=21)$ and Sham-SSA $(n=18)$ rats was similar to $A$, except that rats were not implanted with an intravenous catheter after food training, and locomotor testing occurred after the last day of food training. Rats completed 3 weeks of SSA, followed by progressive-ratio for sucrose pellets while food restricted for $3 \mathrm{~d}$ and while fed ad libitum for $3 \mathrm{~d}$. Rats were then food restricted and restabilized on FR5 timeout $15 \mathrm{~s}$ sucrose self-administration for $3 \mathrm{~d}$. Reinstatement testing consisted of extinction of sucrose-seeking, cue-induced, and sucrose-induced reinstatement testing. C, CSA-WD/IRR $(n=15)$ and CSA-WD/Sham $(n=9)$ rats were irradiated after 3 weeks of $C S A$ and, after 4 weeks of WD coincident with the recovery period, were then tested for reinstatement. All rats were age matched to the beginning of CSA or SSA. Scale bar, $50 \mu \mathrm{m}$. Lightning bolt, $2 \mathrm{~d}$ of $10 \mathrm{~Gy}$ cranial irradiation, in a 1-cm-diameter circle on the dorsal surface of the head to target the hippocampus as described previously (Snyder et al., 2005). H, Habituation; FT/S, food training and intravenous catheter surgery; $r$, recovery; sac, sacrifice by intracardial perfusion; Reinstate, reinstatement testing.

Using published protocols to reduce hippocampal neurogenesis in the adult rat via cranial irradiation (Snyder et al., 2005; Winocur et al., 2006) and assess vulnerability to addiction and propensity for relapse (Graham et al., 2007), we tested the hypothesis that reduced adult hippocampal neurogenesis would lead to increased drug-taking and drug-seeking behaviors. We report that reduction of adult hippocampal neurogenesis increases motivation for drug-taking and drug-seeking and enhances resistance to extinction in the drug-taking environment. Thus, diminished hippocampal neurogenesis is a risk factor in an animal model of cocaine addiction.

\section{Materials and Methods}

Animals. One hundred two adult male Sprague Dawley rats (Charles River) were individually housed in a climate-controlled environment on a $12 \mathrm{~h}$ light/dark cycle (lights on at 7:00 A.M.). Rats were acclimated to vivarium conditions for at least 1 week before experimentation. All experiments were performed in accordance with the National Institutes of Health Guide for the Care and Use of Laboratory Animals in an Institutional Animal Care and Use Committee and Association for Assessment and Accreditation of Laboratory Animal Care approved facility at the University of Texas Southwestern Medical Center. All steps were taken to minimize the number of rats used as well as the pain and suffering of the rats.

Experimental overview. As shown in Figure 1 and as described in detail below, rats used for this study were divided among three experiments. First, to examine the effect of focused cranial irradiation (IRR) on co- caine self-administration (CSA), rats received either IRR or sham irradiation before CSA (IRR-CSA, $n=21$; Sham-CSA, $n=18$ ) (Fig. $1 A)$. Second, to examine the effect of IRR on a natural reward, rats received either IRR or sham irradiation before sucrose selfadministration (IRR-SSA, $n=21$; Sham-SSA, $n=18$ ) (Fig. $1 B$ ). Finally, to examine the effect of irradiation on reinstatement behaviors, rats received either IRR or sham irradiation after CSA and 4 weeks of withdrawal (CSA-WD/ IRR, $n=15$; CSA-WD/Sham, $n=9$ ) (Fig. 1C).

Cranial irradiation. We used a cranial irradiation paradigm shown previously to be optimal for irreversibly suppressing adult hippocampal neurogenesis in the rat (Snyder et al., 2005; Winocur et al., 2006). For 2 consecutive days, rats were anesthetized with sodium pentobarbital $(50 \mathrm{mg} / \mathrm{kg}$, i.p.) and exposed to either 0 Gy (Sham rats) or 10 Gy of irradiation (IRR rats; $1.08 \mathrm{~Gy} / \mathrm{min}, 250 \mathrm{kV}, 15 \mathrm{~mA}$ ) via an X-RAD 320 self-contained irradiation system (Precision X-Ray). For IRR rats, irradiation was focused in a 1-cm-diameter circle over the hippocampus [interaural, 8.00 to $-2.00 \mathrm{~mm}$ (Paxinos and Watson, 1997)] to minimize irradiation of other brain regions (e.g., olfactory bulb, rostral migratory stream, and cerebellum). As shown in Figure 1, rats were exposed to sham or cranial IRR at either $37 \mathrm{~d}$ of age (IRR-CSA, Sham-CSA; IRR-SSA, Sham-SSA) or $98 \mathrm{~d}$ of age (CSA-WD/IRR, CSA-WD/ Sham) and allowed 4 weeks of recovery in the home cage before additional experimentation.

Food training and intravenous catheter surgery. To facilitate acquisition of cocaine selfadministration for the four groups of CSA rats (IRR-CSA, Sham-CSA, CSA-WD/IRR, and CSA-WD/Sham) (Fig. 1 $A, C$ ), these rats were placed on a restricted diet $[\sim 15 \mathrm{~g}$ of chow per day (Edwards et al., 2007)]. For the next 3 d, rats were placed in operant chambers (Med Associates) in which they learned to press the active left lever for $45 \mathrm{mg}$ sucrose pellets (Bio-Serv). All rats used for the CSA studies reported here reached acquisition criterion, which was 100 pellets/d for 3 consecutive days. After food training, rats were fed ad libitum at least $1 \mathrm{~d}$ before implantation with a chronic indwelling intravenous catheter as described previously (Noonan et al., 2008). Cocaine self-administration via the intravenous catheter began after 1 week of recovery from the surgery.

Cocaine self-administration. After food training and 1 week after intravenous catheter surgery, IRR-CSA, Sham-CSA, CSA-WD/IRR, and CSA$\mathrm{WD} /$ Sham rats self-administered cocaine intravenously in $4 \mathrm{~h}$ daily sessions for $15 \mathrm{~d}$ (Fig. $1 \mathrm{~A}, C$ ). Cocaine hydrochloride was provided by the National Institute on Drug Abuse (Baltimore, MD). During CSA, the intravenous infusions of cocaine were always (1) paired with the active/ left lever, (2) accompanied by illumination of the cue light above the left lever, (3) delivered in a $0.1 \mathrm{ml}$ volume over $5 \mathrm{~s}$, and (4) followed by a $15 \mathrm{~s}$ timeout during which the cue and house lights were off for the last $10 \mathrm{~s}$. The response requirement for cocaine infusions was steadily increased to facilitate drug-seeking during later reinstatement testing (Keiflin et al., 2008). For example, for days 1-6 of CSA (acquisition phase), rats selfadministered cocaine on a fixed-ratio reinforcement schedule (FR1): one active/left lever press led to a $0.5 \mathrm{mg} / \mathrm{kg}$ intravenous infusion of cocaine. For days 7-8 of CSA, the schedule increased to FR3, requiring three lever presses for an infusion. For days 9-15 of CSA, the schedule increased to FR5, requiring five lever presses for an infusion. Consistent with addiction literature (Richardson and Roberts, 1996; Lu et al., 2003), these are defined as fixed-ratio, rather than progressive-ratio, schedules because there is no "breakpoint" that is reached by the rats during these self- 
administration sessions, as rats press at evenly spaced intervals after the initial loading period.

On day 16 , most IRR-CSA $(n=14)$ and Sham-CSA $(n=13)$ and all CSA-WD/IRR and CSA-WD/Sham rats underwent within-session doseresponse testing. Four doses of cocaine $(1,0.33,0.10$, and $0.03 \mathrm{mg} / \mathrm{kg}$ ) were made available in descending order, as described previously (Graham et al., 2007). The remaining rats from the IRR-CSA $(n=7)$ and Sham-CSA $(n=5)$ groups did not undergo dose-response testing but rather progressive-ratio testing on days $16-21$ as described previously (Fig. $1 \mathrm{~A}$ ), operationally defined as the escalation of response requirement until the subject will no longer respond, having reached a breakpoint. The rats that underwent progressive-ratio testing self-administered one of three doses of cocaine $(0.1,0.25$, and $0.75 \mathrm{mg} / \mathrm{kg})$ in two consecutive daily sessions in counterbalanced dose order over a total of $6 \mathrm{~d}$. The testing followed the progressive-ratio schedule described previously (Richardson and Roberts, 1996), in which each successive infusion required progressively higher lever pressing according to the following series: $1,2,4,6,9,12,15,20,25,32,40,50,62$, etc., according to response requirement $=\left[5 e^{(\text {injection } \# \times 0.2)}\right]-5$. The breakpoint, or highest ratio of responses/infusions completed before a $1 \mathrm{~h}$ lapse in earning infusions, was analyzed using data from the second test at each dose (Graham et al., 2007).

Immediately after the dose-response testing or the last day of progressive-ratio testing, all rats were tested for catheter patency with sodium methohexital $(0.1 \mathrm{mg}$ in $0.1 \mathrm{ml})$. IRR-CSA and Sham-CSA rats that underwent progressive-ratio testing were killed the following day for immunohistochemical analyses. However, to enable reinstatement testing in the remaining IRR-CSA and Sham-CSA rats and in all CSA-WD/ IRR and CSA-WD/Sham rats, these rats received 4 weeks of withdrawal in their home cage after dose-response testing (Fig. $1 A, C$ ). Rats were handled every $3 \mathrm{~d}$ during this period.

Reinstatement testing. After CSA and 4 weeks of withdrawal in the home cage, the remaining IRR-CSA and Sham-CSA and all the CSAWD/IRR and CSA-WD/Sham rats went through $6 \mathrm{~d}$ of reinstatement testing (Graham et al., 2007), followed by $1 \mathrm{~d}$ of locomotor testing. Reinstatement testing involved exposing the rat to stimuli known to induce relapse to drug-seeking (the context or cue light paired previously with CSA, the drug itself, or a stressful stimuli). Lever presses were recorded during the context-, cue-, drug-, and stress-induced reinstatement testing, but presses on the formerly drug-paired left lever were not reinforced with cues or cocaine infusion. Left lever presses were used as a measure of drug-seeking. On day 1, contextual reinstatement was tested in a $4 \mathrm{~h}$ session. For the entire $4 \mathrm{~h}$ session, rats were placed in the selfadministration chambers where they had previously received cocaine. On day 2, cue-induced reinstatement was tested in a $4 \mathrm{~h}$ session. After $3 \mathrm{~h}$ in the self-administration chamber, the cue light above the formerly drug-paired left lever was illuminated for $5 \mathrm{~s}$ every minute over a span of $30 \mathrm{~min}$. On days $3-5$, rats were tested for cocaine-induced reinstatement in $2 \mathrm{~h}$ daily sessions. After $1 \mathrm{~h}$ in the self-administration chamber, rats received an intraperitoneal injection of $0 \mathrm{mg} / \mathrm{kg}$ cocaine on day 3 , and 5 or $15 \mathrm{mg} / \mathrm{kg}$ cocaine on days 4 and 5 in counterbalanced order. On day 6 , stress-induced reinstatement was tested in a $2 \mathrm{~h}$ session. After $1 \mathrm{~h}$ in the self-administration chamber, mild footshocks were delivered intermittently for $30 \mathrm{~min}$ ( $1.0 \mathrm{~mA}$ in $0.5 \mathrm{~s}$ with random intervals averaging $30 \mathrm{~s})$. On day 7, locomotor activity was recorded $90 \mathrm{~min}$ before and after an injection of cocaine ( $15 \mathrm{mg} / \mathrm{kg}$, i.p.) in a circular test chamber as reported previously (Edwards et al., 2007). All rats were killed the following day for immunohistochemical analyses.

Sucrose self-administration and reinstatement testing. To assess the impact of cranial irradiation on a nondrug reward, rats in the IRR-SSA and Sham-SSA groups self-administered sucrose pellets (Fig. $1 B$ ) in a manner as similar as possible to cocaine administration in the IRR-CSA and Sham-CSA groups (Fig. 1A). IRR-SSA and Sham-SSA rats received cranial or sham irradiation as described above and were left in the home cage for 4 weeks. Rats were handled every $3 \mathrm{~d}$ during this period and were placed on food restriction to encourage sucrose pellet selfadministration $1 \mathrm{~d}$ before food training (the last day of the 4 weeks in home cage). Four weeks after irradiation, IRR-SSA and Sham-SSA rats underwent food training on an FR1 schedule to reach an acquisition criterion of 100 pellets per day for 3 consecutive days. Two days after acquisition, and almost 5 weeks after IRR, locomotor activity was measured for $90 \mathrm{~min}$ as described previously (Edwards et al., 2007) to determine whether irradiated rats showed signs of sickness that might impair their ability or motivation to seek food. After food training and locomotor testing, rats self-administered sucrose pellets in $30 \mathrm{~min}$ daily sessions for $15 \mathrm{~d}$ following the escalating FR schedule used for the CSA groups (days 1-6, FR1; days 7-8, FR3; days 9-15, FR5; 15 s timeout after each reinforcement).

Although every effort was made to keep the cocaine selfadministration and sucrose self-administration studies as similar as possible, we analyzed $4 \mathrm{~h}$ cocaine sessions and only $30 \mathrm{~min}$ sucrose sessions to account for the distinct patterns and length of time animals will press for cocaine versus sucrose. Standard sucrose tests are $30 \mathrm{~min}$ or less because rats are sated after $30 \mathrm{~min}$ or 100 pellets (even with food restriction) and thus will respond infrequently for food after that period. In contrast, standard cocaine-self administration will result in discrete phases of intake (loading followed by regulatory phase) (Tornatzky and Miczek, 2000; Specio et al., 2008). Note that rats responded for $\sim 100$ pellets in that $30 \mathrm{~min}$, whereas in cocaine self-administration, it takes $2-4$ $\mathrm{h}$ to receive that many rewards using the $0.5 \mathrm{mg} / \mathrm{kg}$ dose because of titration of desired cocaine blood levels. The dynamics of the first 10-20 min of cocaine self-administration are thus different from the rest of the $4 \mathrm{~h}$ session, and thus data from the first $30 \mathrm{~min}$ of cocaine selfadministration is not equivalent to data from the $30 \mathrm{~min}$ of sucrose self-administration. However, as described in the next section, progressive ratio was used with both CSA and SSA groups to reduce any constraint of satiety on operant conditioning.

After SSA, rats were run for 1 week on progressive-ratio testing (Fig. $1 B)$. Rats were examined in both food-restricted and sated states (Schmelzeis and Mittleman, 1996) to assess potential alterations in the motivation for food or sensitivity to reward devaluation, respectively. On days $1-3$, food-restricted progressive-ratio testing occurred. Rats were then fed ad libitum on day 4, and, on days 5-7, sated progressive-ratio testing occurred. Rats were then food restricted on day 8 and placed back on the FR5 timeout $15 \mathrm{~s}$ schedule of reinforcement for days 9-11 to restabilize lever pressing before reinstatement testing.

After progressive ratio, rats were assessed for reinstatement testing for 1 week (Fig. $1 B$ ). Reinstatement testing involved exposing the rat to stimuli known to induce relapse to sucrose-seeking (the context or cue light paired previously with SSA, or sucrose pellets). Lever presses were recorded during the context-, cue-, and sucrose-induced reinstatement testing, but presses on the formerly drug-paired left lever were not reinforced with cues or sucrose pellet administration. Left lever presses were used as a measure of sucrose-seeking. On day 1, contextual reinstatement of sucrose-seeking was tested during the $4 \mathrm{~h}$ session. Rats were placed in the operant chamber and left lever presses were recorded, but the cue light did not illuminate and no sucrose pellet was delivered. On day 2, cue reinstatement was tested in a $4 \mathrm{~h}$ session. After $3 \mathrm{~h}$ in the selfadministration chamber, the cue light above the formerly sucrose-paired left lever was illuminated for $5 \mathrm{~s}$ every minute over a span of $30 \mathrm{~min}$. On days $3-5$, rats were placed in the self-administration chambers for $1 \mathrm{~h}$ to extinguish lever pressing. Together with days 1, 2, and 6, this allowed a total of six within-session extinction in both the SSA and CSA reinstatement studies (Fig. 1, compare $A, B$ ). On day 6, sucrose reinstatement was tested in a $4 \mathrm{~h}$ session. After $3 \mathrm{~h}$, noncontingent presentation of two sucrose pellets occurred, followed by one sucrose pellet released every minute for $30 \mathrm{~min}$. Stress-induced reinstatement was not performed, because stress does not reinstate sucrose-seeking (Buczek et al., 1999).

Cocaine brain levels assay. To determine whether irradiation altered the blood-brain barrier and thus potentially bioavailability of cocaine, a subset of IRR-SSA $(n=8)$ and Sham-SSA $(n=8)$ rats were injected with $20 \mathrm{mg} / \mathrm{kg}$ of cocaine intraperitoneally $2 \mathrm{~d}$ after the sucrose reinstatement session (10 weeks after irradiation) and decapitated 15 min later. For cocaine extraction and level determination via mass spectroscopy, brains were removed from the skull, the cerebellum was removed, and the forebrain was frozen in ice-cold isopentane. The brains were stored at $-80^{\circ} \mathrm{C}$ until assay of cocaine levels, as described previously (Edwards et al., 2007). One milliliter of the $1: 4$ diluted homogenate ( $0.25 \mathrm{~g}$ of tissue) from 
A

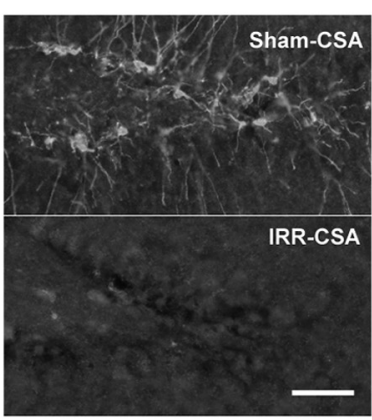

C

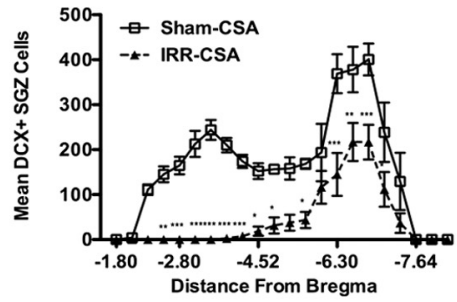

E
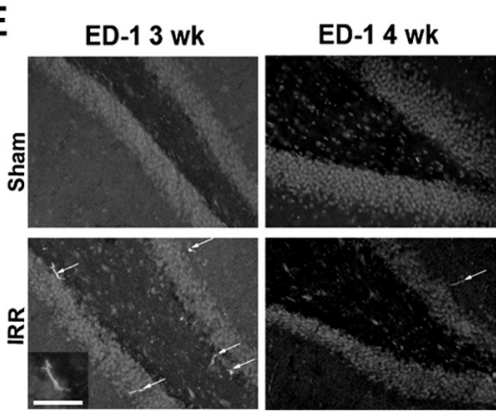

B

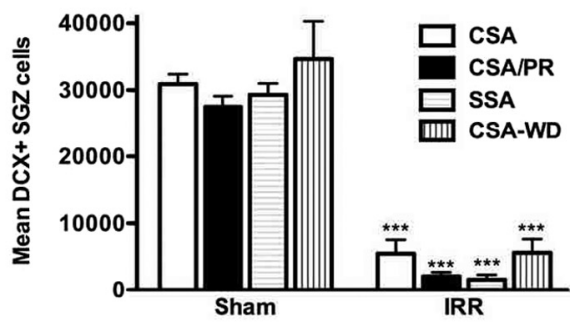

D

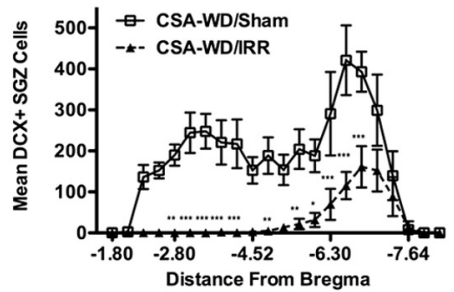

F

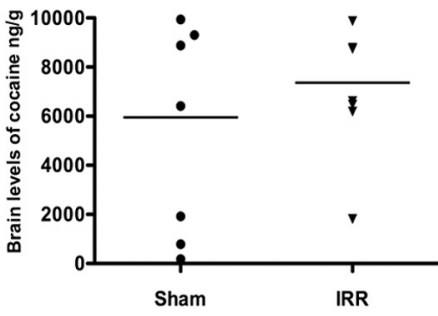

Figure 2. Cranial irradiation produces a long-term decrease in adult hippocampal neurogenesis. $\boldsymbol{A}$, Representative images at $200 \times$ magnification of $\mathrm{DCX}^{+}$immature neurons in the dorsal/anterior SGZ near the apex of the ventral and dorsal dentate gyrus limbs in Sham-CSA and IRR-CSA rats 13 weeks after irradiation. DCX ${ }^{+}$staining was almost completely absent in dorsal dentate gyrus sections in irradiated rats. Scale bar, $50 \mu \mathrm{m}$. B, Quantitative analysis of DCX ${ }^{+}$SGZ cell number at various times after cranial irradiation: CSA, 13 weeks; CSA/progressive-ratio (PR), 9 weeks; SSA, 10 weeks; CSA-WD, 5 weeks. Time line of experiments is provided in Figure 1. Regardless of time after irradiation, the number ofDCX ${ }^{+} S G Z$ cells was significantly reduced relative to control rats, with almost complete ablation of $D C X^{+}$cells in the dorsal dentate gyrus $(\boldsymbol{A})$. C, D, Quantitative analysis of DCX ${ }^{+}$cell number in relation to distance from bregma in Sham-CSA and IRR-CSA rats $(\boldsymbol{C})$ and CSA-WD/Sham and CSA-WD/IRR rats (D). Although $D C X^{+}$staining was almost completely absent in dorsal dentate gyrus sections $(\boldsymbol{A})$, this analysis via distance from bregma reveals that $D C X^{+}$staining was more evident in ventral dentate gyrus sections. This likely represents the loss of radiation energy as it passed from the dorsal to ventral regions of the brain. $\boldsymbol{E}$, Representative photomicrographs from ED1/CD-68-stained sections taken from sham and irradiated rats 3 and 4 weeks after irradiation. Arrows indicate ED1/CD-68 ${ }^{+}$cells in dentate gyrus; inset shows higher magnification of ED1/CD-68 ${ }^{+}$cell to highlight one of the characteristic ramified shape presented by activated microglia. Consistent with previous publications, this paradigm of irradiation resulted in modest microglial activation at 3 weeks. This activation was transient because only rare positive ED1/CD-68 ${ }^{+}$cells were observed at 4 weeks after irradiation and none at later time points (data not shown). Scale bar, $20 \mu \mathrm{m} . \boldsymbol{F}$, Scatter plot of brain levels of cocaine in rats injected with $20 \mathrm{mg} / \mathrm{kg}$ cocaine intraperitoneally. There is no significant difference between the means, and no data points were statistically valid to remove as outliers based on the Grubb's test (Prism; GraphPad Software). Data for $\boldsymbol{B}$ presented as mean \pm SEM. ${ }^{*} p<0.5,{ }^{* *} p<0.01$, *** $p<0.001$

each brain was assayed as follows: $200 \mathrm{ng}$ of deuterated cocaine (Cerilliant) was added as an internal standard, and the $\mathrm{pH}$ was adjusted to 9.3 with ammonium chloride buffer. Cocaine was extracted into $4 \mathrm{ml}$ of $n$-butyl chloride (Fisher Scientific) and subsequently back-extracted into $0.5 \mathrm{ml}$ of $0.1 \mathrm{~N}$ sulfuric acid (Fisher Scientific). The $\mathrm{pH}$ was adjusted back to 9.3 with ammonium chloride buffer, and the cocaine was extracted into $2 \mathrm{ml}$ of $n$-butyl chloride. The dried residue was reconstituted with $30 \mu \mathrm{l}$ of $n$-butyl chloride. Extracts were quantified on an Agilent 5973N GC-MS (Agilent Technologies) in selected ion mode, and results were corrected for deuterated cocaine recovery.

Tissue preparation. All rats except those used in the assay of cocaine brain levels were killed via chloral hydrate anesthesia and intracardial perfusion with $0.1 \mathrm{M}$ PBS (5 min with $10 \mathrm{ml} /$ min flow rate) and 4\% paraformaldehyde in $0.1 \mathrm{M}$ PBS (20 min) as described previously (Noonan et al., 2008). After perfusion, brains were removed and postfixed in $4 \%$ paraformaldehyde in $0.1 \mathrm{M}$ PBS for $24 \mathrm{~h}$ at $4^{\circ} \mathrm{C}$. Brains were cryoprotected in $30 \%$ sucrose in $0.1 \mathrm{M}$ PBS with $0.1 \% \mathrm{NaN}_{3}$ at $4^{\circ} \mathrm{C}$ until coronal sectioning on a freezing microtome (Leica) at $30 \mu \mathrm{m}$ through the entire hippocampus $(-1.80$ to $-7.64 \mathrm{~mm}$ from bregma) (Paxinos and Watson, 1997) and the olfactory bulb (Noonan et al., 2008). Sections were stored in $0.1 \% \mathrm{NaN}_{3}$ in $0.1 \mathrm{M} \mathrm{PBS}$ at $4^{\circ} \mathrm{C}$ until processed for immunohistochemistry (IHC).

IHC and quantification of immunopositive cells. IHC was performed as described previously (Noonan et al., 2008). Briefly, every ninth section of the hippocampus was mounted on glass slides (Superfrost/Plus; Fisher Scientific) and dried overnight. Slides were coded before IHC, and the code was not broken until after microscopic and data analyses were complete. To verify previous work that this irradiation paradigm suppressed indices of hippocampal neurogenesis (Snyder et al., 2005; Winocur et al., 2006; Wojtowicz and Kee, 2006; Airan et al. 2007; Wojtowicz et al., 2008) and produced modest transient inflammation (Wojtowicz and Kee, 2006; Airan et al., 2007), separate series of hippocampal sections were stained with antibodies against the immature neuron marker doublecortin (DCX) (Brown et al., 2003) and the activated microglia marker ED1/ CD68 (Rola et al., 2006; Borders et al., 2007). For DCX and ED1/CD-68 IHC, antigen unmasking $\left(0.01 \mathrm{M}\right.$ citric acid, $\mathrm{pH} 6.0,95^{\circ} \mathrm{C}, 15$ $\mathrm{min}$ ) and quenching of endogenous peroxidases $\left(0.3 \% \mathrm{H}_{2} \mathrm{O}_{2}, 30 \mathrm{~min}\right)$ were performed, and sections were placed into blocking solution for $1 \mathrm{~h}$ ( $3 \%$ serum), followed by primary antibody incubation [goat anti-doublecortin, 1:3000 (Santa Cruz Biotechnology); mouse anti-ED1, 1:500 (AbD Serotec) overnight at room temperature. Sections were then incubated with a biotinylated secondary antibody (horse anti-goat for DCX, donkey anti-mouse for ED1, 1:200; Vector Laboratories), followed by incubation with avidin-biotin (ABC Elite, 1:50; Vector Laboratories). Immunoreactive $(+)$ cells were visualized via FITC tyramide signal amplification (PerkinElmer Life and Analytical Sciences), and 4',6-diamidino-2-phenylindole was used as a nuclear counterstain (1:5000; Roche). Sections underwent rapid dehydration in ethanols and defatting in Citrosolv (Fisher Scientific) before coverslipping with DPX (SigmaAldrich). Although these DCX and ED1 antibodies are widely used and have been shown to lack staining in knock-out animals and provide a single band via immunoblotting, specificity of staining was also ensured by lack of signal after omission of primary antibody and by observation of expected subcellular localization and cellular populations.

Using the optical fractionator method, $\mathrm{DCX}^{+}$cells in the SGZ were counted at $400 \times$ magnification with an Olympus BX-51 microscope while continually adjusting the focal plane through the depth of the section (Noonan et al., 2008). An observer blind to treatment group performed all cell counts. Exhaustive counts were collected from every ninth hippocampal section throughout the anteroposterior extent of the 
hippocampus $[-1.80$ to $-7.64 \mathrm{~mm}$ from bregma (Paxinos and Watson, 1997)]. Resulting cell counts were multiplied by the fraction of the hippocampus examined (e.g., 9) (Noonan et al., 2008) and are reported as total number of cells in the dentate gyrus. ED1/CD68-stained sections were examined by an observer blind to treatment group to gain qualitative information on the presence or absence of activated microglia (Fig. 2).

Statistical analyses and presentation. Data are presented as mean \pm SEM. Statistical analyses used SPSS version 11.0 for Mac. Selfadministration and extinction data were analyzed using two-factor ANOVA with repeated measures on test session. Reinstatement data were analyzed using two- or three-factor ANOVA with repeated measures on dose or test session and lever. $\mathrm{DCX}^{+}$cell counts were analyzed with two-factor ANOVA. Main-effect analyses were followed with Bonferroni's post hoc tests. For analyses with one variable, such as latency to extinction, a $t$ test was used. Outlier analysis on cocaine brain levels was performed via the Grubb's outlier test, but no data points were removed because results were negative. Statistical significance for main effects, interactions, and $t$ tests was defined as $p<0.05$. Images were imported into Photoshop version 9.0.2 (Adobe Systems), and the only adjustments made were via gamma in the levels function.

\section{Results}

Cranial irradiation produced long-term ablation of adult hippocampal neurogenesis without changing brain levels of cocaine

We explored the role of adult hippocampal neurogenesis in cocaine self-administration by giving rats cranial or sham irradiation 5 weeks before self-administration (Fig. 1). As shown previously (Snyder et al., 2005), two consecutive sessions of 10 Gy of cranial irradiation directed over the hippocampus was sufficient to robustly decrease adult hippocampal neurogenesis, as measured by $\mathrm{DCX}^{+}$cell number in the SGZ (Fig. 2 A). The cranial irradiation-induced reduction in neurogenesis was long lasting, because rats examined between 5 and 13 weeks after radiation all showed at least a $70 \%$ reduction in $\mathrm{DCX}^{+} \mathrm{SGZ}$ cell number compared with controls (main effect of treatment, $F_{(1,33)}$ $=213.05, p<0.001$ ) (Fig. $2 B$ ), with almost complete ablation in the dorsal and anterior dentate gyrus and a robust decrease in the ventral and posterior dentate gyrus (Fig. 2C). Although some activated microglia (labeled by ED1/CD-68 antibody) were present in the dentate gyrus of irradiated rats when examined 3 weeks later, this inflammatory effect was modest and transient (Fig. 2E). This is consistent with previous reports that this irradiation paradigm resulted in minimal or at most transient inflammation (Wojtowicz and Kee, 2006; Airan et al., 2007).

To assess whether irradiation changed brain bioavailability of cocaine, we used mass spectrometry to measure brain levels of cocaine after a single cocaine injection given 10 weeks after irradiation. Brain levels of cocaine (nanograms per gram of brain) were similar between control and irradiated rats (Sham, $5956.38 \pm 1526.27$; IRR, $7365.00 \pm 974.87 ; p=0.45$ ) (Fig. $2 F$ ). These data suggest that the blood-brain barrier and the bioavailability of cocaine are not grossly influenced by cranial irradiation
B

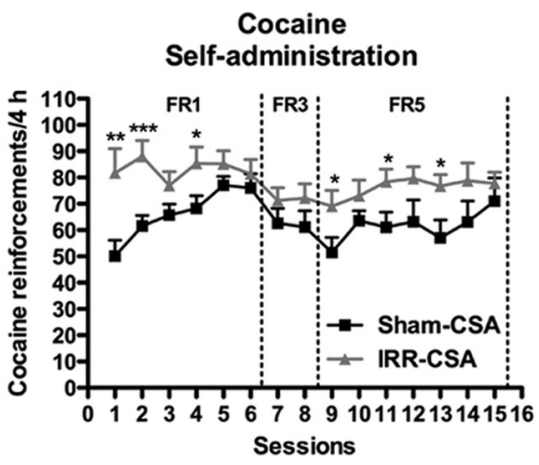

D

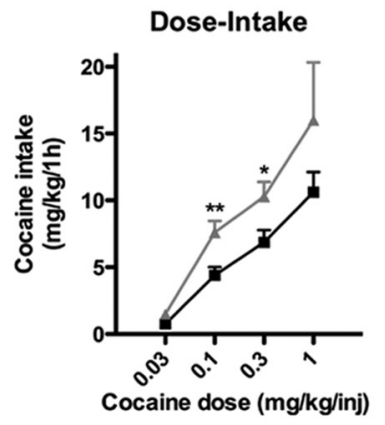

E

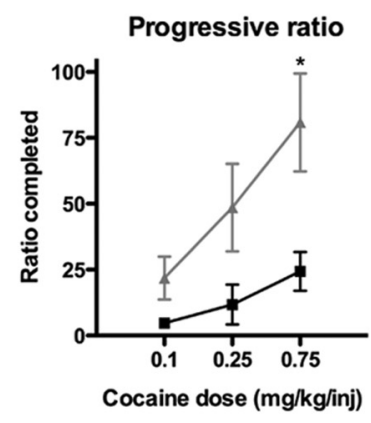

Figure 3. Cranial irradiation before cocaine self-administration increases cocaine reward. $\boldsymbol{A}$, Irradiated rats did not differ from sham rats in the time it took to obtain 100 sucrose pellets during acquisition of an FR1 schedule during food training. $\boldsymbol{B}$, Crania E. I Irradiated rats worked harder to get their last infusion of cocaine, suggesting that they find cocaine more rewarding. Data are presented as mean \pm SEM. ${ }^{*} p<0.05,{ }^{* *} p<0.01,{ }^{* *} p<0.001$.

and that the cocaine self-administration experiments are not confounded by differences in uptake of cocaine into the brain.

\section{Irradiation before cocaine self-administration increased} motivation for cocaine

To address the hypothesis that reduced adult hippocampal neurogenesis is a vulnerability factor for addiction, rats were irradiated (IRR-CSA) or sham-irradiated (Sham-CSA) before cocaine self-administration (Fig. 1A). IRR-CSA rats did not differ from Sham-CSA rats in food training, because the latency to acquire lever pressing for 100 sucrose pellets across sessions was similar (no effect of treatment, $F_{(1,111)}=0.038, p=0.846$ ) (Fig. $3 A$ ). These data suggest that irradiation did not disrupt basic operant learning. However, IRR-CSA rats self-administered more cocaine across 15 daily 4 h sessions (main effect of treatment, $F_{(1,518)}=8.35, p<0.01$ ) (Fig. $3 B$ ). These data show that cranial irradiation, which is accompanied by a robust decrease in hippocampal neurogenesis (Fig. 2), leads to increased selfadministration of cocaine.

Because lesions of the hippocampus produce hyperactivity (Emerich and Walsh, 1990; Tani et al., 2001; Hernández-Rabaza et al., 2008) as well as increase perseverative lever pressing for both water and cocaine self-administration (Rabe and Haddad, 1968; Chambers and Self, 2002), we addressed the possibility that, like a hippocampal lesion, reduced neurogenesis via cranial irradiation might lead to hyperactivity and perseverative responding. Indeed, IRR-CSA rats pressed significantly more on the drugpaired left lever during the $15 \mathrm{~s}$ timeout in the first two selfadministration sessions (interaction of treatment and day, $\left.F_{(14,518)}=1.72, p<0.05\right)($ Fig. $4 A)$, suggesting that greater co- 
A

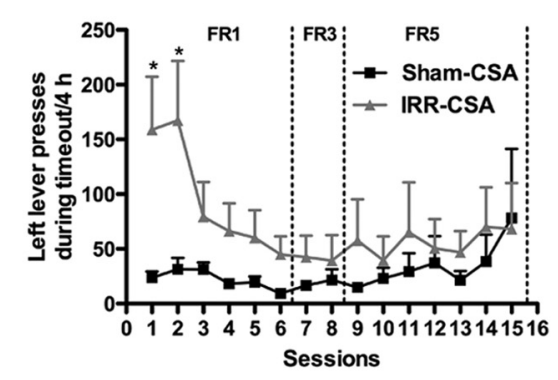

C

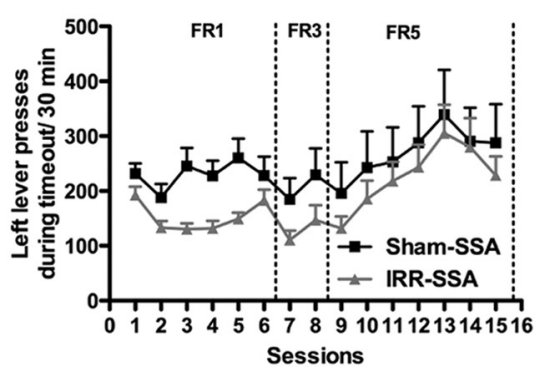

B

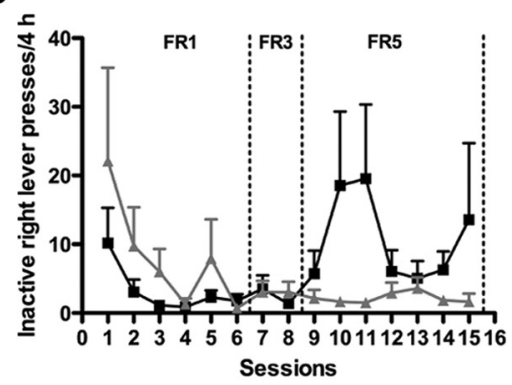

D

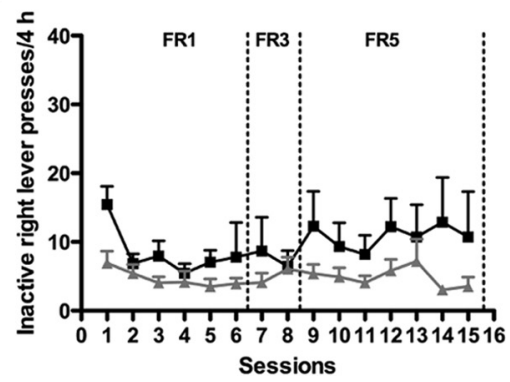

Figure 4. Cranial irradiation transiently increases impulsive responding during the timeout period of cocaine self-administration but does not alter timeout pressing or inactive right lever pressing during sucrose self-administration. $\boldsymbol{A}, \boldsymbol{B}$, Left/active lever presses $(\boldsymbol{A})$ and right/inactive lever presses $(\boldsymbol{B})$ during the $15 \mathrm{~s}$ timeout per daily cocaine self-administration session. Irradiated rats pressed significantly more on the drug-paired lever during the timeout on the first two self-administration sessions but thereafter did not press significantly more than sham rats $(\boldsymbol{A})$. Irradiated rats did not significantly press more on the inactive lever than sham rats during any session $(\boldsymbol{B})$. C, $\boldsymbol{D}$, Left/active lever presses $(\boldsymbol{C})$ and right/inactive lever presses $(\boldsymbol{D})$ during the $15 \mathrm{~s}$ timeout per daily sucrose self-administration session. Irradiated and sham rats pressed the same amount on the food-paired lever $(\boldsymbol{C})$ and the inactive lever $(\boldsymbol{D})$ during the timeout period. Data are presented as mean \pm SEM. ${ }^{*} p<0.05$.

Table 1. Correlations between $\mathrm{DCX}{ }^{+}$immature neuron number and behaviors

\begin{tabular}{lll}
\hline & CSA & CSA-WD \\
\hline Days $1-5$ cocaine intake & 0.007 & \\
Days $11-15$ cocaine intake & 0.002 & \\
PR at $0.1 \mathrm{mg} / \mathrm{kg}$ & 0.022 & \\
PR at $0.75 \mathrm{mg} / \mathrm{kg}$ & $0.386^{*}$ & \\
Extinction latency & 0.004 & 0.096 \\
Cue reinstatement & 0.111 & $0.383^{*}$ \\
Cocaine at $5 \mathrm{mg} / \mathrm{kg}$ reinstatement & 0.315 & 0.002 \\
Footshock reinstatement & 0.054 & 0.009 \\
\hline
\end{tabular}

$R^{2}$ are reported separately for correlations between $D C X^{+}$immature neuron number and self-administration and reinstatement behaviors. $n=24$ for CSA; $n=12$ CSA progressive-ratio and reinstatement correlations; $n=11$ for all CSA-WD correlations. * $p<0.05$. PR, Progressive ratio.

caine intake during the first and second self-administration sessions is likely attributable to perseveration. Perseveration did not occur on the subsequent 13 daily self-administration sessions, and there was no difference between IRR-CSA and Sham-CSA rats in inactive right lever pressing on any of the 15 daily sessions (no effect of treatment, $F_{(1,518)}=0.27, p=0.61$ ) (Fig. $4 B$ ). Together, these data suggest that the overall increase in cocaine intake was not attributable to hyperactivity or perseverative behavior. Together with the fact that irradiation did not alter bioavailability of cocaine in the brain, we then explored other hypotheses as to why irradiated rats self-administered more cocaine.

First, we tested the hypothesis that cranial irradiation led to greater cocaine self-administration because the rats were more sensitive to the reinforcing effects of cocaine. To this end, rats underwent a within-session dose-response test the day after the 15 th self-administration session. IRR-CSA rats had a vertical shift in the dose-response curve (interaction of treatment and dose, $F_{(3,75)}=3.14, p<$ 0.05 ) (Fig. 3C). This vertical shift is indicative of increased sensitivity to cocaine and is considered a standard measure of the "addicted" phenotype in laboratory animals (Piazza et al., 2000). When the number of infusions is multiplied by the amount of cocaine infused, IRR-CSA rats took more cocaine at several doses (interaction of treatment and dose, $F_{(3,75)}=3.76, p<0.05$ ) (Fig. 3D).

Second, we extended our exploration of the impact of cranial irradiation on the reinforcing aspects of cocaine via progressive-ratio testing, which assesses how many lever presses a rat is willing to perform for a single infusion of cocaine (Richardson and Roberts, 1996). To this end, a subset of IRR-CSA and ShamCSA rats received progressive-ratio testing instead of dose-response testing. IRR-CSA rats worked harder than Sham-CSA rats to receive their last infusion of cocaine (main effect of treatment, $\left.F_{(1,20)}=5.51, p<0.05\right)$ (Fig. $3 E$ ). These data indicate that cocaine was more reinforcing for irradiated rats and rule out the possibility that rats took more cocaine because they were less sensitive to cocaine. Interestingly, we found a significant correlation between $\mathrm{DCX}^{+}$cell number in IRR-CSA and Sham-CSA rats and breakpoints on the progressive ratio testing at $0.75 \mathrm{mg} / \mathrm{kg}$ (Table 1). Because progressive-ratio testing is a sensitive assay for motivation, this suggests a direct effect of levels of neurogenesis on motivation for cocaine reward.

Together, the fixed-ratio, dose-response, and progressiveratio data suggest that rats that have been cranially irradiated and have reduced neurogenesis are more motivated to take cocaine as well as seek cocaine and thus can be defined as more vulnerable to drug addiction (Piazza et al., 2000).

\section{Irradiation before sucrose self-administration does not change motivation for sucrose}

To address whether suppression of adult hippocampal neurogenesis increased motivation for all reinforcers or only for drugs of abuse, we used a natural reward: sucrose self-administration. Rats were irradiated (IRR-SSA) or sham-irradiated (Sham-SSA) before sucrose self-administration (Fig. $1 B$ ). Latency to acquire lever pressing for 100 sucrose pellets was not different between groups (no effect of treatment, $F_{(1,111)}=0.38, p=0.54$ ) (Fig. $5 A$ ), confirming data from the CSA group that irradiation does not interfere with learning of an operant task. Furthermore, locomotor activity assessed 5 weeks after irradiation was not different between groups (no effect of treatment, $F_{(1,629)}=0.05, p=0.82$ ) (Fig. $5 B$ ), suggesting that irradiation studies were not confounded by locomotor impairments. Sucrose self-administration on an FR1, FR3, or FR5 schedule with a $15 \mathrm{~s} \mathrm{timeout} \mathrm{did} \mathrm{not} \mathrm{differ}$ between groups (no main effect of treatment, $F_{(1,518)}=2.04, p=$ 0.16 ) (Fig. 5C). Both IRR-SSA and Sham-SSA rats were similar in left lever pressing during the $15 \mathrm{~s}$ timeout (no effect of treatment, $F_{(1,518)}=1.87, p=0.18$ ) (Fig. $4 C$ ) and pressing on the right 
A

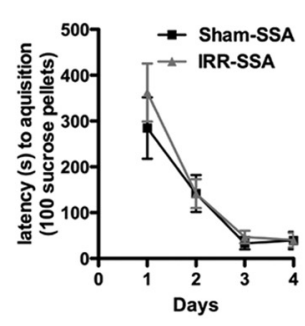

D

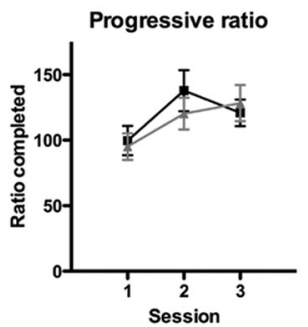

B

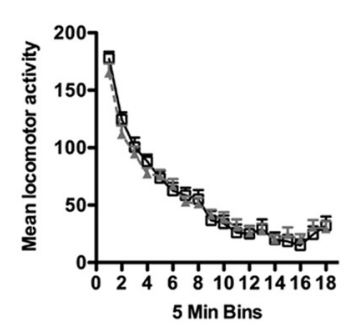

E

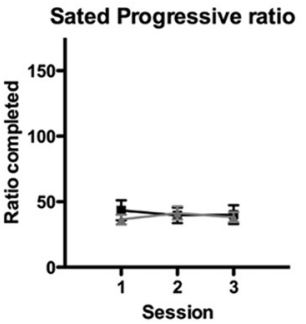

F
C
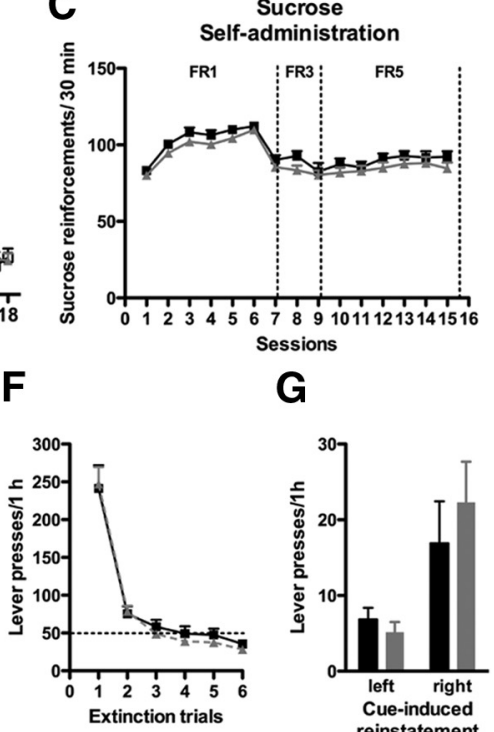

G

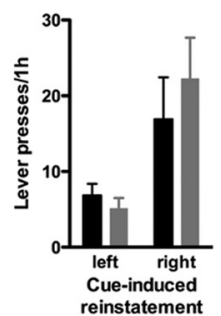

H

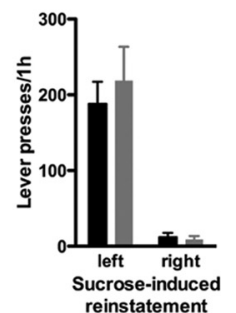

Figure 5. Cranial irradiation before sucrose self-administration does not change natural reward. $A$, Irradiated rats did not differ from sham rats in the time it took to obtain 100 sucrose pellets during acquisition of an FR1 schedule during food training. $\boldsymbol{B}$, Basal locomotion was not different between irradiated and sham rats. $\boldsymbol{C}$, Irradiated rats were not different from sham rats in sucrose pellet self-administration on an FR1, FR3, or FR5 schedule. $\boldsymbol{D}$, $\boldsymbol{E}$, Sucrose pellet self-administration on a progressive ratio was not different between irradiated and sham rats when rats were food restricted during testing days $(\boldsymbol{D})$ or when not food restricted $(\boldsymbol{E})$. $\boldsymbol{F}$, Irradiated rats did not differ from sham rats in their ability to extinguish responding on the formerly sucrose-paired lever. $\boldsymbol{G}$, Irradiated rats did not differ from sham rats in responding to cues formerly paired with sucrose administration. $\boldsymbol{H}$, Irradiated rats did not differ from sham rats in responding to presentation of sucrose pellets. Data are presented as mean \pm SEM.

inactive lever (no effect of treatment, $F_{(1,518)}=2.61, p=0.12$ ) (Fig. 4D), suggesting that their general activity was the same. These data suggest that self-administration of food, a natural reward, was not altered by irradiation, and irradiated rats do not suffer from lack of appetite or motor impairment.

To confirm that irradiation did not alter motivation for food, we used a more sensitive assay that would be less influenced by satiety, progressive-ratio testing, while on and off food restriction. IRR-SSA rats worked as hard as Sham-SSA rats to receive their last sucrose pellet reward (no effect of treatment, $F_{(1,74)}=$ $0.10, p=0.75$ ) (Fig. $5 D$ ), suggesting that sucrose was equally reinforcing for both groups. When food restriction was removed, both groups were less willing to work for their last sucrose pellet reward than when they were food restricted (no effect of treatment, $F_{(1,74)}=0.10, p=0.75$ ) (Fig. $5 E$ ), suggesting equal sensitivity to reward devaluation. Together with the fixed-ratio data, these data suggest that irradiated rats do not have altered motivation for sucrose.

We next tested the ability of irradiated rats to learn that pressing on the left lever no longer was reinforced with sucrose in a series of extinction tests, as well as their ability to relapse to sucrose-seeking when presented with sucrose-paired cues or sucrose itself. Both IRR-SSA and Sham-SSA rats extinguished pressing on the formerly sucrose-paired lever (no effect of treatment, $\left.F_{(1,185)}=0.15, p=0.70\right)$ (Fig. $5 F$ ). There was no difference between groups in pressing on the formerly sucrose-paired lever in response to presentation of sucrose-paired cues (no effect of treatment, $F_{(1,37)}=0.20, p=0.66$ ) (Fig. $5 G$ ) or sucrose pellets (no effect of treatment, $F_{(1,37)}=0.22, p=0.65$ ) (Fig. $5 H$ ).

In summary, irradiated rats with reduced hippocampal neurogenesis have normal motivation for natural reward, because rats show no difference in self-administration of sucrose, extinction of sucrose-seeking, or relapse to sucrose-seeking after reexposure to sucrose-related cues or sucrose itself. In addition, these experiments addressed possible confounds of the irradiation be- fore cocaine-self administration experiment. Irradiated rats did not show perseveration, general increased activity, or general learning enhancement when response requirements are increased (i.e., an increase from FR3 to FR5 schedule or the progressive-ratio task) and were able to learn to extinguish responding when reinforcement was withheld, suggesting that any changes seen in motivation for reward after irradiation are not a result of experimental confounds.

\section{Irradiation after cocaine self-administration enhanced resistance to extinction}

Having identified that rats with suppressed adult neurogenesis are more vulnerable to acquisition of drug addiction, we next addressed the hypothesis that suppression of adult neurogenesis during withdrawal increases vulnerability to relapse to drugseeking. To this end, rats were irradiated (CSA-WD/IRR) or sham-irradiated (CSA-WD/Sham) after acquisition of cocaine self-administration (Fig. 1C). As with irradiation before CSA (Fig. 2C), irradiation after CSA led to ablation of $\mathrm{DCX}^{+}$cells in the dorsal and anterior dentate gyrus and a robust decrease in $\mathrm{DCX}^{+}$cell number in the ventral and posterior dentate gyrus (Fig. 2D).

CSA-WD/Sham and CSA-WD/IRR rats were assigned to these groups by balanced cocaine self-administration (no main effect of treatment, $F_{(1,308)}=0.05, p=0.83$ ) (Fig. 6A). After a 1 month withdrawal period, rats went through reinstatement testing to assess their vulnerability to relapse to drug-seeking. CSA-WD/ IRR rats pressed more on the formerly drug-paired lever when they were reexposed to the former drug-taking context each day (interaction of treatment and session, $F_{(5,18)}=2.99, p<0.05$ ) (Fig. $6 \mathrm{~B}$ ) and took more trials to extinguish their drug-seeking $(p<0.01)$ (Fig. 6C). Both groups were similar in lever pressing in response to formerly drug-paired cues (no main effect of treatment, $F_{(1,22)}=3.79, p=0.06$ ) (Fig. $6 D$ ) and low-dose cocaine 
A
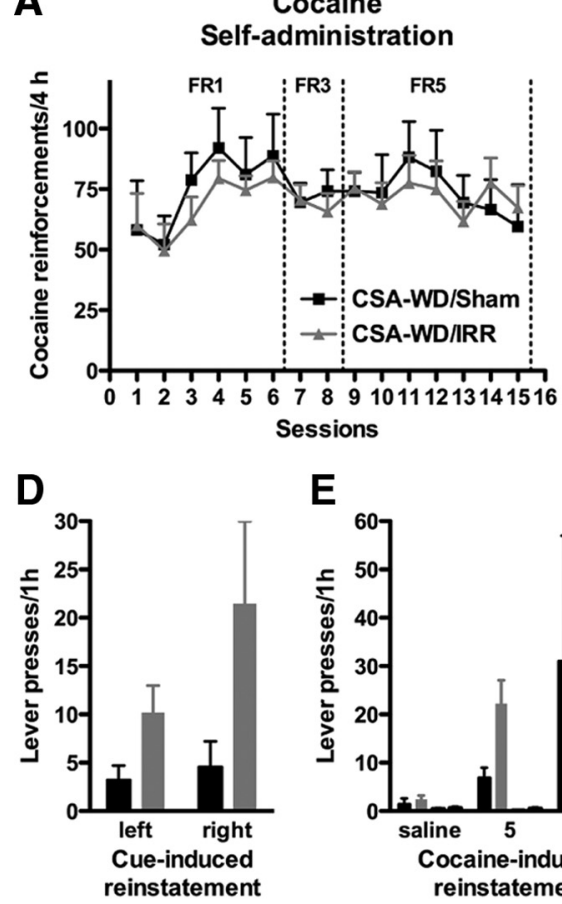

E

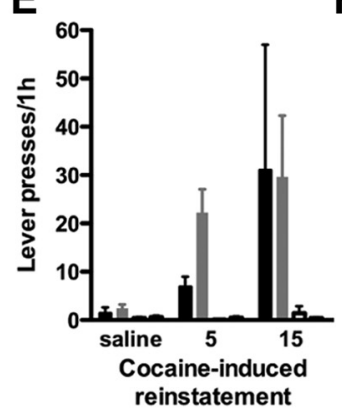

B

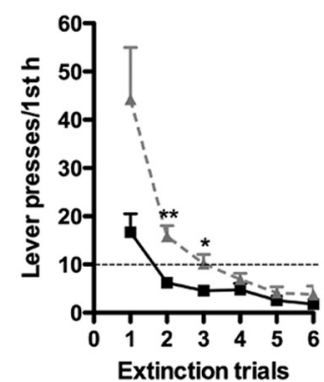

$\mathbf{F}$

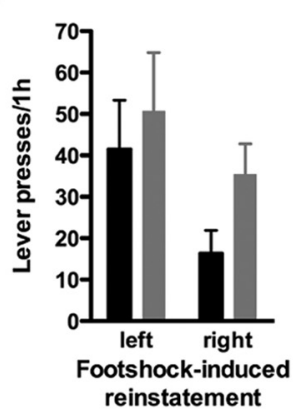

C

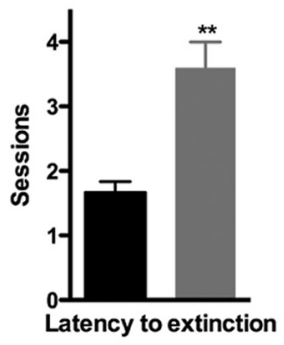

G

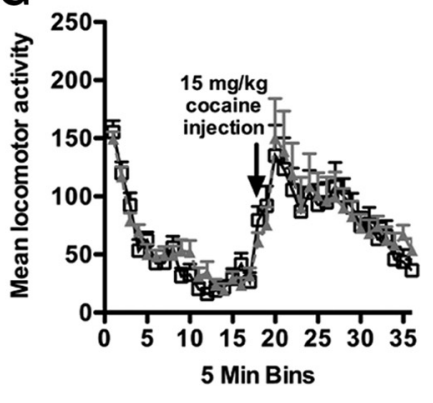

Figure 6. Cranial irradiation after cocaine self-administration enhances resistance to extinction. $A$, Self-administration of cocaine. Cocaine self-administration in each group before irradiation. Rat were then divided to make two statistically similar groups and assigned to CSA-WD/IRR or CSA-WD/Sham groups. B, C, Context-induced reinstatement. Irradiation after CSA lead to enhanced context-dependent reinstatement. Irradiated rats pressed more on the formerly drug-paired lever $(\boldsymbol{B})$ and took more sessions to extinguish lever pressing on the formerly drug-paired lever $(\boldsymbol{C})$ when reexposed daily to the self-administration chamber in the absence of reinforcement after 4 weeks of abstinence. $\boldsymbol{D}$, Cue-induced reinstatement. Irradiated rats had a nonstatistical trend toward pressing both the formerly drug-paired lever and the inactive lever more than sham-irradiated rats in response to drug cues. $E$, Drug-induced reinstatement. Response to pressing the left, formerly drug-paired lever (leftmost pair of bars) or right, formerly drug-unpaired lever (rightmost pair of bars) after intraperitoneal injection of saline or 5 or $15 \mathrm{mg} / \mathrm{kg}$ cocaine in sham and irradiated rats. Irradiated rats had a nonstatistical trend toward pressing the formerly drug-paired left lever more in response to low-dose $(5 \mathrm{mg} / \mathrm{kg}$, i.p.) cocaine exposure. Right lever presses are presented but are under five for both irradiated and sham groups at every dose of cocaine. $\boldsymbol{F}$, Footshock stress-induced reinstatement. Lever pressing between irradiated and sham-irradiated rats in response to footshock stress. G, Locomotion assessment. Irradiated rats were not impaired in either basal locomotion or cocaine-induced locomotion (15 mg/kg, i.p.). Data are presented as mean \pm SEM. ${ }^{*} p<0.05,{ }^{* *} p<0.01$.

injections (no main effect of treatment, $F_{(1,44)}=0.28, p=0.60$ ) (Fig. 6E), although there were trends toward significance for both cue- and drug-induced $(5 \mathrm{mg} / \mathrm{kg})$ reinstatement. CSA-WD/IRR rats did not differ from sham rats in lever pressing in response to footshock stress (no main effect of treatment, $F_{(1,22)}=1.26, p=$ 0.27 ) (Fig. $6 F$ ), suggesting that irradiation does not alter the hypothalamic-pituitary-adrenal (HPA) axis and response to stress in this paradigm. Both groups had similar basal and cocaineinduced locomotion (no main effect of treatment, $F_{(1,374)}=0.23$, $p=0.88)($ Fig. $6 G)$, suggesting that increased extinction responding was not attributable to hyperactivity. These data suggest decreased neurogenesis increases vulnerability to relapse after exposure to the former drug-taking environment.

Interestingly, although we found no correlation between $\mathrm{DCX}^{+}$cell number and responding on a variety of measures, including the cue-induced reinstatement test in IRR-CSA and Sham-CSA rats (Table 1), we did find a significant correlation between $\mathrm{DCX}^{+}$cell number in CSA-WD/IRR and CSA-WD/ Sham rats and responding on the cue-induced reinstatement test (Table 1). This suggests that the timing of neurogenesis modulation as well as the levels of neurogenesis are important for response to drug-associated cues. It is not surprising that levels of hippocampal neurogenesis correlates with cue-induced reinstatement of cocaine-seeking, because the hippocampus has been shown to be necessary for the task (Rogers and See, 2007).
Rats irradiated before cocaine self-administration did not have altered extinction or reinstatement responding

We next addressed the hypothesis that reduced hippocampal neurogenesis during both cocaine self-administration and withdrawal would increase relapse to drug-seeking. Rats were irradiated or sham irradiated 1 month before initial cocaine selfadministration and went through reinstatement testing after 1 month of abstinence (Fig. 1A). Because rats that have increased cocaine intake also have greater relapse to drug-seeking (Sutton et al., 2000; Edwards et al., 2007) and rats irradiated after cocaine self-administration (CSA-WD/IRR) had increased resistance to extinction (Fig. 6B,C), we expected IRR-CSA rats to show increased extinction and reinstatement test responding. However, irradiated rats did not differ in extinction of pressing on the formerly drug-paired lever (no effect of treatment, $F_{(1,125)}=0.85$, $p=0.36$ ) (Fig. 7A). Irradiated rats did not differ from sham rats in lever pressing in response to formerly drug-paired cues (no effect of treatment, $F_{(1,25)}=0.33, p=0.57$ ) (Fig. $7 B$ ) or in response to low-dose cocaine injections (no effect of treatment, $\left.F_{(1,50)}=0.63, p=0.43\right)$ (Fig. $7 C$ ). Additionally, irradiated rats did not differ from sham rats in lever pressing in response to footshock stress (no effect of treatment, $F_{(1,25)}=1.82, p=0.19$ ) (Fig. 7D). Finally, irradiated rats did not differ from sham rats in basal locomotion or cocaine-induced hyperlocomotion (no effect of treatment, $F_{(1,425)}=0.01, p=0.89$ ) (Fig. $7 E$ ), suggesting that the lack of differences in drug-seeking in irradiated rats is not 
attributable to nonspecific side effects of irradiation. These data suggest that reduced neurogenesis before cocaine selfadministration does not increase later drug-seeking, despite increasing cocaine intake during self-administration before the withdrawal period (Fig. 3B).

\section{Discussion}

Our first notable finding is that cranial irradiation before drug-taking enhanced vulnerability in an animal model of cocaine addiction. Irradiated rats (IRR-CSA) had increased cocaine self-administration under fixed- and progressive-ratio schedules compared with controls. This increase was not caused by irradiation-induced changes in response to a novel environment or operant learning, increased locomotion, perseveration of active lever pressing, or unequal brain levels of cocaine between sham and irradiated rats. Intriguingly, sucrose self-administration was not changed after irradiation (IRR-SSA). Given that addictive drugs as diverse as opiates, ethanol, and stimulants result in common neuroadaptations and behaviors (Nestler, 2005), it will be important to explore whether suppression of hippocampal neurogenesis enhances vulnerability to self-administration of other drugs of abuse.

Our second notable finding is that cranial irradiation during the forced abstinence period after acquisition of cocaine self-administration (CSA-WD/IRR) increased the propensity for contextdependent relapse. This deficit in extinction fits with other recent work showing that ablation of neurogenesis disrupts extinction learning of spatial preference and contextual fear (Deng et al., 2009) and highlights the novel role uncovered here for adultgenerated neurons in addictive processes. Although care must be taken in comparing extinction from drug self-administration versus from context learning, there are helpful principles from the latter that help interpret the present results. For example, of the three aspects of extinction-acquisition, consolidation, and retrieval (Quirk and Mueller, 2008) - it is unlikely that CSA$\mathrm{WD} / \mathrm{IRR}$ rats have a deficit in acquisition because they were able to reduce responding within extinction sessions and their latency to the last lever press was not different from sham rats (data not shown). The hippocampus plays a role in extinction consolidation and retrieval (Cammarota et al., 2005; Corcoran et al., 2005), impeding distinction of which aspect is altered in the present study. However, by assessing discrete behavioral features of extinction (Ji and Maren, 2007), future studies can dissect whether contextual stimuli elicited higher levels of responding in CSA$\mathrm{WD} / \mathrm{IRR}$ than sham rats attributable to deficits in retrieval or consolidation.

Previously, we found that CSA-WD increased SGZ immature neuron number (Noonan et al., 2008), and we hypothesized that adult-generated hippocampal neurons contributed to drugcraving or relapse. However, our CSA-WD/IRR data show re-
B
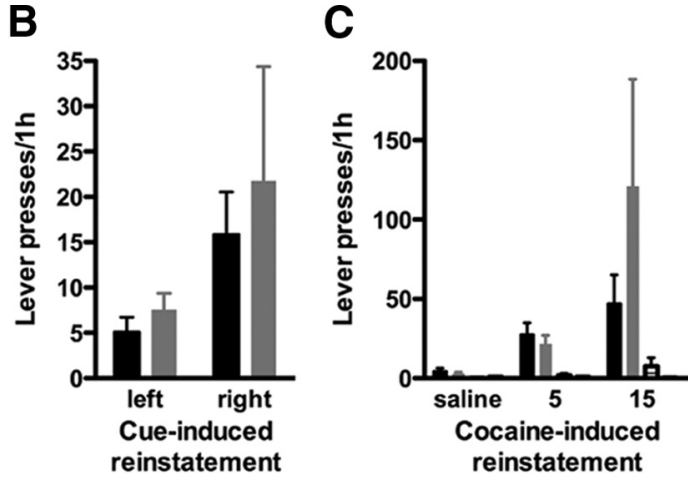

E

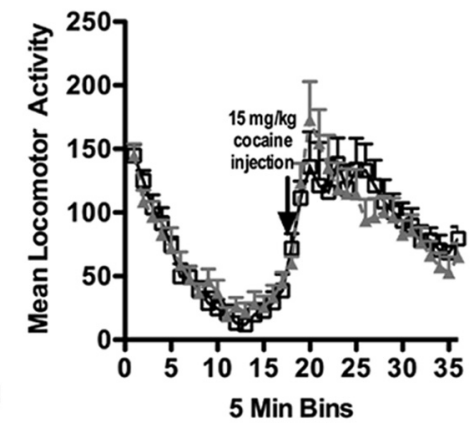

reinstatement

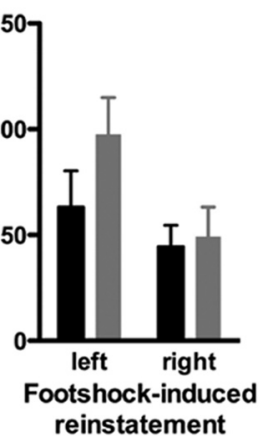

Figure 7. Cranial irradiation before cocaine self-administration does not change relapse to drug-seeking. $\boldsymbol{A}$, Context-induced reinstatement. Irradiated rats did not press more than sham rats on the formerly drug-paired lever when reexposed to the (intration chamber after 4 weeks of abstinence. $\boldsymbol{B}$, Cue-induced reinstatement. Irradiated rats did not differ from sham lever (leftmost pair of bars) or right, formerly drug-unpaired lever (rightmost pair of bars) after intraperitoneal injection of saline of cocaine. D, Footshock stress-induced reinstatement. Lever pressing between irradiated and sham-irradiated rats in response to footshock stress was not significantly different. $\boldsymbol{E}$, Locomotion assessment. Irradiated rats were not impaired in either basal locomotion or cocaine-induced locomotion (15 mg/kg, i.p.). Data are presented as mean \pm SEM.

duced hippocampal neurogenesis enhanced relapse to drugseeking. Thus, the CSA-WD-induced increase in immature neurons appears beneficial. This clarified interpretation of our previous results also fits with our finding that suppression of adult hippocampal neurogenesis is a vulnerability factor in this animal model of addiction. Because manipulations that enhance neurogenesis can diminish the positive-reinforcing effects of cocaine (Smith et al., 2008) and suppression of neurogenesis can impair extinction outside of the context of addiction (Deng et al., 2009), it will be interesting to examine whether manipulations that further increase neurogenesis during the withdrawal period facilitate extinction of CSA.

Although suppression of neurogenesis after cocaine selfadministration (CSA-WD/IRR) enhanced drug-seeking and thus produced extinction deficits, cranial irradiation before cocaine self-administration (IRR-CSA) did not increase cocaine-seeking in the former drug-taking context after abstinence. This was surprising because rats that self-administer more cocaine have increased drug-seeking (Sutton et al., 2003; Edwards et al., 2007), but this is consistent with recent work that reduction of neurogenesis does not influence extinction (Ko et al., 2009). Why is extinction altered when neurogenesis is reduced after, but not before, CSA? Adult-generated neurons encode part of the longterm contextual memory (Snyder et al., 2001; Imayoshi et al., 2008), and cells in distinct stages of adult neurogenesis support discrete aspects of learning and memory (Dupret et al., 2007). 
Therefore, it is likely meaningful that IRR-CSA rats lacked adultgenerated neurons during both acquisition and the withdrawal period, whereas CSA-WD/IRR rats only lacked them during withdrawal. Also, IRR-CSA rats lacked adult-generated neurons for a longer period of time and so perhaps were able to compensate for the loss of the "plasticity reserve" proposed to be provided by adult-generated neurons (Kempermann, 2008).

\section{Irradiation-induced suppression of hippocampal neurogenesis, and not other side effects, mediates altered cocaine-taking and cocaine-seeking}

There are several reasons we used cranial irradiation for this study. Cranial irradiation reduces hippocampal neurogenesis to as great or greater extent, in a shorter period of time, and with equal or greater consistency compared with alternative methods (Dupret et al., 2005; Saxe et al., 2006; Imayoshi et al., 2008). Our focused cranial irradiation paradigm provides a relatively restricted suppression hippocampal neurogenesis. Mitotic inhibitors and most transgenic approaches also suppress neurogenesis in the olfactory bulb, which could independently increase sensitivity to psychostimulants (Holmes et al., 2002; Slattery et al., 2007). Finally, focused cranial irradiation did not reduce locomotion, induce sickness, or require additional minipump surgery (Dupret et al., 2005; Saxe et al., 2006). These factors made cranial irradiation the preferred method to launch these fundamental studies on the relationship between adult hippocampal neurogenesis and cocaine self-administration.

Based on our study and many others that use similar irradiation paradigms to decrease rat hippocampal neurogenesis (Snyder et al., 2005; Winocur et al., 2006; Wojtowicz and Kee, 2006; Airan et al., 2007; Wojtowicz et al., 2008), this paradigm maintains the integrity of mature hippocampal neurons while ablating neural progenitors. It did not grossly damage the dentate gyrus, because global hippocampal morphology and locomotion were not affected (Emerich and Walsh, 1990; Tani et al., 2001; Hernández-Rabaza et al., 2008). This paradigm does not alter electrophysiological properties of mature hippocampal neurons or network dynamics (Snyder et al., 2005; Airan et al., 2007), suggesting that irradiation-induced alterations in hippocampal function are primarily attributable to the loss of adult-generated neurons (Wojtowicz, 2006). Irradiation decreased the weight gain of rats in all groups but did not cause weight loss, consistent with previous reports (Snyder et al., 2005), suggesting that rats maintained their food intake after irradiation. Gross alteration in HPA axis function, which enhances vulnerability to addiction (Deroche et al., 1997), is also unlikely because irradiated rats did not have altered footshock stress-induced reinstatement, which is influenced by HPA function (Erb et al., 1998). Increased drugtaking and drug-seeking is likely not secondary to the development of anxiety or other symptoms relevant to mood disorders, because rats were normal on many activity measures and irradiation-induced reduction of adult hippocampal neurogenesis does not induce symptoms of depression (Santarelli et al., 2003; Airan et al., 2007; Surget et al., 2008). Irradiation did not grossly compromise the vasculature, because brain levels of cocaine were not different between sham and irradiated rats, and testing began 4 weeks after irradiation, a period after which inflammation is minimal (Fig. 2E) (Snyder et al., 2005; Wojtowicz, 2006; Airan et al., 2007). Future studies irradiating alternative or "control" brain regions would help rule out remaining concerns, including the impact of residual inflammation or factors secreted from activated microglia (Kohl et al., 2007). However, together with previous work on irradiation, our data suggest that irradiation-induced reduction of adult hippocampal neurogenesis, and not side effects of irradiation, are responsible for increased drug-taking and drug-seeking.

\section{Possible mechanism of control over cocaine-taking and cocaine-seeking by adult hippocampal neurogenesis}

Increased drug-taking and drug-seeking in irradiated rats with reduced adult hippocampal neurogenesis fits with the role of the dentate gyrus as an inhibitory filter for hippocampal activity (Hsu, 2007). Activating or inactivating the subiculum, which provides the glutamatergic projections from the hippocampus to the nucleus accumbens and other limbic regions (Kelley and Domesick, 1982; Pitkanen et al., 2000; Herman and Mueller, 2006), increases or decreases drug-taking and drug-seeking, respectively (Caine et al., 2001; Vorel et al., 2001; Fuchs et al., 2005). If adult-generated neurons are indeed functionally similar to embryonic-generated ones (Laplagne et al., 2006), it possible that decreasing the number of adult-generated dentate gyrus neurons results in hippocampal disinhibition, leading to increased activation of hippocampus-accumbens-ventral pallidum-ventral tegmental area circuitry (Floresco et al., 2001). This can increase dopamine release from the ventral tegmental area in response to cocaine or the cocaine context and may underlie behavioral sensitization to stimulants (Lodge and Grace, 2008) and raises a reasonable hypothesis: ablation of adult neurogenesis may disinhibit neural circuitry responsible for cocaine-taking.

Although this hypothesis implies that adult-generated dentate gyrus neurons function similarly to embryonic or postnatally generated neurons in regards to drug-taking and drug-seeking, they may also be functionally distinct. Temporary inactivation of the hippocampus blocks context-induced, cue-induced, and cocaine-induced reinstatement of cocaine-seeking (Fuchs et al., 2005; Rogers and See, 2007), which is opposite to our results in which irradiation after cocaine withdrawal enhanced contextinduced reinstatement. Thus, adult-generated hippocampal neurons may have a different and an unappreciated role in specific addiction-relevant behaviors relative to older granule cell neurons. Functional studies comparing recruitment of adult-generated versus embryonic or early postnatally generated neurons into addiction circuitry will be necessary to determine whether this is the case.

\section{Possible clinical relevance}

These studies suggest that reduced adult hippocampal neurogenesis may enhance the transition from recreation to addiction and facilitate drug relapse, especially when faced with a former drugtaking environment. Treatments that increase adult neurogenesis may prevent addiction before it starts, which would be especially important for patients treated with potentially addictive medications. Additionally, treatments that increase adult neurogenesis during abstinence may prevent relapse.

\section{References}

Abrous DN, Adriani W, Montaron MF, Aurousseau C, Rougon G, Le Moal M, Piazza PV (2002) Nicotine self-administration impairs hippocampal plasticity. J Neurosci 22:3656-3662.

Abrous DN, Koehl M, Le Moal M (2005) Adult neurogenesis: from precursors to network and physiology. Physiol Rev 85:523-569.

Airan RD, Meltzer LA, Roy M, Gong Y, Chen H, Deisseroth K (2007) Highspeed imaging reveals neurophysiological links to behavior in an animal model of depression. Science 317:819-823.

Baker DA, Tran-Nguyen TL, Fuchs RA, Neisewander JL (2001) Influence of individual differences and chronic fluoxetine treatment on cocaineseeking behavior in rats. Psychopharmacology (Berl) 155:18-26. 
Borders AS, Getchell ML, Etscheidt JT, van Rooijen N, Cohen DA, Getchell TV (2007) Macrophage depletion in the murine olfactory epithelium leads to increased neuronal death and decreased neurogenesis. J Comp Neurol 501:206-218.

Brown J, Cooper-Kuhn CM, Kempermann G, Van Praag H, Winkler J, Gage FH, Kuhn HG (2003) Enriched environment and physical activity stimulate hippocampal but not olfactory bulb neurogenesis. Eur J Neurosci 17:2042-2046.

Buczek Y, Lê AD, Wang A, Stewart J, Shaham Y (1999) Stress reinstates nicotine seeking but not sucrose solution seeking in rats. Psychopharmacology (Berl) 144:183-188.

Caine SB, Humby T, Robbins TW, Everitt BJ (2001) Behavioral effects of psychomotor stimulants in rats with dorsal or ventral subiculum lesions: locomotion, cocaine self-administration, and prepulse inhibition of startle. Behav Neurosci 115:880-894.

Cammarota M, Bevilaqua LR, Barros DM, Vianna MR, Izquierdo LA, Medina JH, Izquierdo I (2005) Retrieval and the extinction of memory. Cell Mol Neurobiol 25:465-474.

Chambers RA, Self DW (2002) Motivational responses to natural and drug rewards in rats with neonatal ventral hippocampal lesions: an animal model of dual diagnosis schizophrenia. Neuropsychopharmacology 27:889-905.

Corcoran KA, Desmond TJ, Frey KA, Maren S (2005) Hippocampal inactivation disrupts the acquisition and contextual encoding of fear extinction. J Neurosci 25:8978-8987.

Covington HE 3rd, Miczek KA (2005) Intense cocaine self-administration after episodic social defet stress, but not after aggressive behavior: dissociation from corticosterone activation. Psychopharmacology (Berl) 183: 331-340.

Deng W, Saxe MD, Gallina IS, Gage FH (2009) Adult-born hippocampal dentate granule cells undergoing maturation modulate learning and memory in the brain. J Neurosci 29:13532-13542.

Deroche V, Marinelli M, Le Moal M, Piazza PV (1997) Glucocorticoids and behavioral effects of psychostimulants. II. Cocaine intravenous selfadministration and reinstatement depend on glucocorticoid levels. J Pharmacol Exp Ther 281:1401-1407.

Dupret D, Montaron MF, Drapeau E, Aurousseau C, Le Moal M, Piazza PV, Abrous DN (2005) Methylazoxymethanol acetate does not fully block cell genesis in the young and aged dentate gyrus. Eur J Neurosci 22:778-783.

Dupret D, Fabre A, Döbrössy MD, Panatier A, Rodríguez JJ, Lamarque S, Lemaire V, Oliet SH, Piazza PV, Abrous DN (2007) Spatial learning depends on both the addition and removal of new hippocampal neurons. PLoS Biol 5:e214.

Dupret D, Revest JM, Koehl M, Ichas F, De Giorgi F, Costet P, Abrous DN, Piazza PV (2008) Spatial relational memory requires hippocampal adult neurogenesis. PLoS One 3:e1959.

Edwards S, Whisler KN, Fuller DC, Orsulak PJ, Self DW (2007) Addictionrelated alterations in $\mathrm{d}(1)$ and $\mathrm{d}(2)$ dopamine receptor behavioral responses following chronic cocaine self-administration. Neuropsychopharmacology 32:354-366.

Eisch AJ (2002) Adult neurogenesis: implications for psychiatry. Prog Brain Res 138:315-342.

Eisch AJ, Barrot M, Schad CA, Self DW, Nestler EJ (2000) Opiates inhibit neurogenesis in the adult rat hippocampus. Proc Natl Acad Sci U S A 97:7579-7584.

Emerich DF, Walsh TJ (1990) Hyperactivity following intradentate injection of colchicine: a role for dopamine systems in the nucleus accumbens. Pharmacol Biochem Behav 37:149-154.

Erb S, Shaham Y, Stewart J (1996) Stress reinstates cocaine-seeking behavior after prolonged extinction and drug-free period. Psychopharmacology (Berl) 128:408-412.

Erb S, Shaham Y, Stewart J (1998) The role of corticotropin-releasing factor and corticosterone in stress- and cocaine-induced relapse to cocaine seeking in rats. J Neurosci 18:5529-5536.

Floresco SB, Todd CL, Grace AA (2001) Glutamatergic afferents from the hippocampus to the nucleus accumbens regulate activity of ventral tegmental area dopamine neurons. J Neurosci 21:4915-4922.

Fuchs RA, Evans KA, Ledford CC, Parker MP, Case JM, Mehta RH, See RE (2005) The role of the dorsomedial prefrontal cortex, basolateral amygdala, and dorsal hippocampus in contextual reinstatement of cocaine seeking in rats. Neuropsychopharmacology 30:296-309.

Graham DL, Edwards S, Bachtell RK, DiLeone RJ, Rios M, Self DW (2007)
Dynamic BDNF activity in nucleus accumbens with cocaine use increases self-administration and relapse. Nat Neurosci 10:1029-1037.

Green TA, Gehrke BJ, Bardo MT (2002) Environmental enrichment decreases intravenous amphetamine self-administration in rats: dose-response functions for fixed- and progressive-ratio schedules. Psychopharmacology (Berl) 162:373-378.

Herman JP, Mueller NK (2006) Role of the ventral subiculum in stress integration. Behav Brain Res 174:215-224.

Hernández-Rabaza V, Hontecillas-Prieto L, Velázquez-Sánchez C, Ferragud A, Pérez-Villaba A, Arcusa A, Barcia JA, Trejo JL, Canales JJ (2008) The hippocampal dentate gyrus is essential for generating contextual memories of fear and drug-induced reward. Neurobiol Learn Mem 90:553-559.

Holmes PV, Masini CV, Primeaux SD, Garrett JL, Zellner A, Stogner KS, Duncan AA, Crystal JD (2002) Intravenous self-administration of amphetamine is increased in a rat model of depression. Synapse 46:4-10.

Hsu D (2007) The dentate gyrus as a filter or gate: a look back and a look ahead. Prog Brain Res 163:601-613.

Hubbard RL, Marsden ME (1986) Relapse to use of heroin, cocaine, and other drugs in the first year after treatment. NIDA Res Monogr 72:157-166.

Imayoshi I, Sakamoto M, Ohtsuka T, Takao K, Miyakawa T, Yamaguchi M, Mori K, Ikeda T, Itohara S, Kageyama R (2008) Roles of continuous neurogenesis in the structural and functional integrity of the adult forebrain. Nat Neurosci 11:1153-1161.

Ji J, Maren S (2007) Hippocampal involvement in contextual modulation of fear extinction. Hippocampus 17:749-758.

Kanarek RB, Marks-Kaufman R, D’Anci KE, Przypek J (1995) Exercise attenuates oral intake of amphetamine in rats. Pharmacol Biochem Behav 51:725-729.

Keiflin R, Vouillac C, Cador M (2008) Level of operant training rather than cocaine intake predicts level of reinstatement. Psychopharmacology (Berl) 197:247-261.

Kelley AE, Domesick VB (1982) The distribution of the projection from the hippocampal formation to the nucleus accumbens in the rat: an anterograde- and retrograde-horseradish peroxidase study. Neuroscience 7:2321-2335.

Kempermann G (2008) The neurogenic reserve hypothesis: what is adult hippocampal neurogenesis good for? Trends Neurosci 31:163-169.

Ko HG, Jang DJ, Son J, Kwak C, Choi JH, Ji YH, Lee YS, Son H, Kaang BK (2009) Effect of ablated hippocampal neurogenesis on the formation and extinction of contextual fear memory. Mol Brain 2:1.

Kohl Z, Kandasamy M, Winner B, Aigner R, Gross C, Couillard-Despres S, Bogdahn U, Aigner L, Winkler J (2007) Physical activity fails to rescue hippocampal neurogenesis deficits in the R6/2 mouse model of Huntington's disease. Brain Res 1155:24-33.

Laplagne DA, Espósito MS, Piatti VC, Morgenstern NA, Zhao C, van Praag H, Gage FH, Schinder AF (2006) Functional convergence of neurons generated in the developing and adult hippocampus. PLoS Biol 4:e409.

Lodge DJ, Grace AA (2008) Amphetamine activation of hippocampal drive of mesolimbic dopamine neurons: a mechanism of behavioral sensitization. J Neurosci 28:7876-7882.

Lu L, Shepard JD, Hall FS, Shaham Y (2003) Effect of environmental stressors on opiate and psychostimulant reinforcement, reinstatement and discrimination in rats: a review. Neurosci Biobehav Rev 27:457-491.

Malberg JE, Eisch AJ, Nestler EJ, Duman RS (2000) Chronic antidepressant treatment increases neurogenesis in adult rat hippocampus. J Neurosci 20:9104-9110.

Mandyam CD, Wee S, Crawford EF, Eisch AJ, Richardson HN, Koob GF (2008) Varied access to intravenous methamphetamine self-administration differentially alters adult hippocampal neurogenesis. Biol Psychiatry 64:958-965.

Meyers RA, Zavala AR, Speer CM, Neisewander JL (2006) Dorsal hippocampus inhibition disrupts acquisition and expression, but not consolidation, of cocaine conditioned place preference. Behav Neurosci 120:401-412.

Miczek KA, Mutschler NH (1996) Activational effects of social stress on IV cocaine self-administration in rats. Psychopharmacology (Berl) 128:256-264.

Mirescu C, Gould E (2006) Stress and adult neurogenesis. Hippocampus 16:233-238

Nestler EJ (2005) Is there a common molecular pathway for addiction? Nat Neurosci 8:1445-1449.

Noonan MA, Choi KH, Self DW, Eisch AJ (2008) Withdrawal from cocaine 
self-administration normalizes deficits in proliferation and enhances maturity of adult-generated hippocampal neurons. J Neurosci 28:2516-2526.

Paxinos GT, Watson C (1997) The rat brain in stereotaxic coordinates compact third edition. New York: Academic.

Piazza PV, Deroche-Gamonent V, Rouge-Pont F, Le Moal M (2000) Vertical shifts in self-administration dose-response functions predict a drugvulnerable phenotype predisposed to addiction. J Neurosci 20:4226-4232.

Pitkänen A, Pikkarainen M, Nurminen N, Ylinen A (2000) Reciprocal connections between the amygdala and the hippocampal formation, perirhinal cortex, and postrhinal cortex in rat. A review. Ann N Y Acad Sci 911:369-391.

Quirk GJ, Mueller D (2008) Neural mechanisms of extinction learning and retrieval. Neuropsychopharmacology 33:56-72.

Rabe A, Haddad RK (1968) Effect of selective hippocampal lesions in the rat on acquisition, performance, and extinction of bar pressing on a fixed ratio schedule. Exp Brain Res 5:259-266.

Reif A, Fritzen S, Finger M, Strobel A, Lauer M, Schmitt A, Lesch KP (2006) Neural stem cell proliferation is decreased in schizophrenia, but not in depression. Mol Psychiatry 11:514-522.

Richardson NR, Roberts DC (1996) Progressive ratio schedules in drug selfadministration studies in rats: a method to evaluate reinforcing efficacy. J Neurosci Methods 66:1-11.

Rogers JL, See RE (2007) Selective inactivation of the ventral hippocampus attenuates cue-induced and cocaine-primed reinstatement of drug-seeking in rats. Neurobiol Learn Mem [Erratum (2008) 89:86] 87:688-692.

Rola R, Mizumatsu S, Otsuka S, Morhardt DR, Noble-Haeusslein LJ, Fishman K, Potts MB, Fike JR (2006) Alterations in hippocampal neurogenesis following traumatic brain injury in mice. Exp Neurol 202:189-199.

Santarelli L, Saxe M, Gross C, Surget A, Battaglia F, Dulawa S, Weisstaub N, Lee J, Duman R, Arancio O, Belzung C, Hen R (2003) Requirement of hippocampal neurogenesis for the behavioral effects of antidepressants. Science 301:805-809.

Saxe MD, Battaglia F, Wang JW, Malleret G, David DJ, Monckton JE, Garcia AD, Sofroniew MV, Kandel ER, Santarelli L, Hen R, Drew MR (2006) Ablation of hippocampal neurogenesis impairs contextual fear conditioning and synaptic plasticity in the dentate gyrus. Proc Natl Acad Sci U S A 103:17501-17506.

Schmelzeis MC, Mittleman G (1996) The hippocampus and reward: effects of hippocampal lesions on progressive-ratio responding. Behav Neurosci 110:1049-1066.

Shen F, Meredith GE, Napier TC (2006) Amphetamine-induced place preference and conditioned motor sensitization requires activation of tyrosine kinase receptors in the hippocampus. J Neurosci 26:11041-11051.

Slattery DA, Markou A, Cryan JF (2007) Evaluation of reward processes in an animal model of depression. Psychopharmacology (Berl) 190:555-568.

Smith MA, Schmidt KT, Iordanou JC, Mustroph ML (2008) Aerobic exercise decreases the positive-reinforcing effects of cocaine. Drug Alcohol Depend 98:129-135.
Snyder JS, Kee N, Wojtowicz JM (2001) Effects of adult neurogenesis on synaptic plasticity in the rat dentate gyrus. J Neurophysiol 85:2423-2431.

Snyder JS, Hong NS, McDonald RJ, Wojtowicz JM (2005) A role for adult neurogenesis in spatial long-term memory. Neuroscience 130:843-852.

Specio SE, Wee S, O’Dell LE, Boutrel B, Zorrilla EP, Koob GF (2008) CRF(1) receptor antagonists attenuate escalated cocaine self-administration in rats. Psychopharmacology (Berl) 196:473-482.

Stairs DJ, Klein ED, Bardo MT (2006) Effects of environmental enrichment on extinction and reinstatement of amphetamine self-administration and sucrose-maintained responding. Behav Pharmacol 17:597-604.

Surget A, Saxe M, Leman S, Ibarguen-Vargas Y, Chalon S, Griebel G, Hen R, Belzung C (2008) Drug-dependent requirement of hippocampal neurogenesis in a model of depression and of antidepressant reversal. Biol Psychiatry 64:293-301.

Sutton MA, Karanian DA, Self DW (2000) Factors that determine a propensity for cocaine-seeking behavior during abstinence in rats. Neuropsychopharmacology 22:626-641.

Sutton MA, Schmidt EF, Choi KH, Schad CA, Whisler K, Simmons D, Karanian DA, Monteggia LM, Neve RL, Self DW (2003) Extinction-induced upregulation in AMPA receptors reduces cocaine-seeking behaviour. Nature 421:70-75.

Tani K, Iyo M, Matsumoto H, Kawai M, Suzuki K, Iwata Y, Won T, Tsukamoto T, Sekine Y, Sakanoue M, Hashimoto K, Ohashi Y, Takei N, Mori N (2001) The effects of dentate granule cell destruction on behavioural activity and Fos protein expression induced by systemic methamphetamine in rats. Br J Pharmacol 134:1411-1418.

Tornatzky W, Miczek KA (2000) Cocaine self-administration "binges": transition from behavioral and autonomic regulation toward homeostatic dysregulation in rats. Psychopharmacology (Berl) 148:289-298.

United Nations Office on Drugs and Crime (2008) World drug report. In: United Nations Office on Drugs and Crime. New York: United Nations Publications.

van Praag H, Kempermann G, Gage FH (1999) Running increases cell proliferation and neurogenesis in the adult mouse dentate gyrus. Nat Neurosci 2:266-270.

Vorel SR, Liu X, Hayes RJ, Spector JA, Gardner EL (2001) Relapse to cocaine-seeking after hippocampal theta burst stimulation. Science 292: $1175-1178$.

Winocur G, Wojtowicz JM, Sekeres M, Snyder JS, Wang S (2006) Inhibition of neurogenesis interferes with hippocampus-dependent memory function. Hippocampus 16:296-304.

Wojtowicz JM (2006) Irradiation as an experimental tool in studies of adult neurogenesis. Hippocampus 16:261-266.

Wojtowicz JM, Kee N (2006) BrdU assay for neurogenesis in rodents. Nat Protoc 1:1399-1405.

Wojtowicz JM, Askew ML, Winocur G (2008) The effects of running and of inhibiting adult neurogenesis on learning and memory in rats. Eur J Neurosci 27:1494-1502. 